\title{
Assessment of streetcar transit priority options using microsimulation modelling ${ }^{1}$
}

\author{
Amer Shalaby, Baher Abdulhai, and Jinwoo Lee
}

\begin{abstract}
The thrust of a recently published transportation vision for Toronto is focused largely on reducing automobile dependence via a number of interacting strategies, including the wide application of transit priority policies to improve transit competitiveness. This paper reports on quantifying the impacts of several transit priority schemes, with the streetcar operation along King Street in the heart of Toronto as a case study. Four scenarios were modelled in a microsimulation framework. They include the status quo (involving unconditional transit signal priority, already in operation), turning off existing transit signal priority, prohibiting all left turns, and finally prohibiting traffic from King Street. To quantify the impacts of any of the above scenarios, a set of common measures of effectiveness was used, including transit travel time and speed, effective headway, service frequency and person throughput, bunching, fleet size implications, and overall traffic and transit average speeds. The results show the relative merits of the four scenarios and two strategies for improving streetcar service along the King route are recommended. The first is to prohibit all left turns along the route, while the second, admittedly more aggressive, is to potentially transform the arterial into a transit mall accessible only to streetcars.
\end{abstract}

Key words: transit signal priority, transit priority, signal control, microsimulation, streetcars.

Résumé : L'orientation d'une vision du transport public de Toronto, récemment publiée, porte principalement sur la réduction de la dépendance vis à vis l'automobile par l'utilisation d'une variété de stratégies interdépendantes, incluant l'application étendue de politiques de priorité de passage afin d'améliorer la compétitivité du service de transport public. Cet article quantifie les impacts de plusieurs schémas de priorité de passage en utilisant comme étude de cas le tramway le long de la rue King, au cœur de Toronto. Quatre scénarios ont été modélisés dans le cadre d'une microsimulation. Ils comprennent le statu quo (impliquant un signal de priorité de passage sans condition, déjà en opération), arrêter les signaux de priorité de passage existants, interdire tous les virages à gauche et enfin interdire toute circulation sur la rue King. Afin de quantifier les impacts de chacun de ces scénarios, un ensemble de mesures communes d'efficacité a été utilisé; celui-ci comprenait le temps et la vitesse des véhicules de transport public, l'intervalle efficace, la fréquence du service aux usagers et le nombre de personnes, le regroupement (bunching), les implications de la taille de la flotte, ainsi que les vitesses moyennes globales de la circulation et des véhicules du transport public. Les résultats montrent les mérites relatifs des quatre scénarios et recommande deux stratégies pour améliorer le service de tramway le long du parcours King. La première recommandation est d'interdire tous les virages à gauche le long du parcours alors que la seconde, qu'il faut reconnaître plus audacieuse, est de transformer possiblement cette artère en une rue réservée uniquement aux piétons et aux tramways.

Mots clés : signal de priorité de passage, priorité de passage, commande des signaux, microsimulation, tramways.

[Traduit par la Rédaction]

\section{Introduction}

A recently published transportation "vision" is intended to complement the new official plan for the amalgamated City

Received 31 July 2002. Revision accepted 12 February 2003.

Published on the NRC Research Press Web site at

http://cjce.nrc.ca on 2 September 2003.

A. Shalaby, ${ }^{2}$ B. Abdulhai, and J. Lee. Intelligent

Transportation Systems Centre and Testbed, Department of

Civil Engineering, University of Toronto, 35 St. George

Street, Toronto, ON M5S 1A4, Canada.

Written discussion of this article is welcomed and will be received by the Editor until 30 April 2004.

${ }^{1}$ This article is one of a selection of papers published in this Special Issue on Innovations in Transportation Engineering.

${ }^{2}$ Corresponding author (e-mail: amer@ecf.utoronto.ca). of Toronto (City of Toronto 2001). The thrust of the vision is focused largely on reducing automobile dependence via a number of interacting strategies, including enhancing the competitiveness of public transit relative to the private automobile.

To translate the vision statements and principles into reality, a course of action was recommended that included an indepth study of the potential benefits of transit priority as an essential step towards improving the attractiveness of public transit throughout the City of Toronto. The vision report identified transit travel time as one of the most serious competitive disadvantages relative to the private automobile. A significant percentage of transit delays occur at intersections, which may be blocked by cross street traffic and (or) automobile turning movements. Such delays lead to lower average speeds of transit vehicles and consequently to poorer service at higher cost. The vision recommended a more aggressive application of transit priority policies to improve 
transit competitiveness, while admittedly realizing that such improvements can only be achieved at some cost to other road users. This study was launched to analyze and quantify such impacts.

\section{Study scope and approach}

In consultation with the City of Toronto and the Toronto Transit Commission (TTC), the 504 King streetcar route (Fig. 1) was chosen for this study. Beginning at the Dundas West subway station and heading south, the route stretches along Dundas Street West, Roncesvalles Ave., King Street, a short section of Queen Street East, and Broadview Ave., ending at the Broadview subway station. All crossroads up to (but not including) the next intersection were included in the study area.

Four scenarios were focused on, including the status quo (which involves unconditional transit signal priority already in operation), the termination of existing transit signal priority, the continuation of transit priority provision while restricting vehicular traffic left turns, and finally the transformation of the arterial into a transit mall, banning all traffic but streetcars. The second scenario is examined so as to assess the performance of the streetcar route without the unconditional transit signal priority now in operation (i.e., to measure the effectiveness of the existing priority scheme).

\section{The integrated transit-traffic microsimulation approach}

This section presents a brief overview of the microsimulation approach this study used for the integrated modelling of transit and traffic operations in the 504 King streetcar corridor.

\section{The simulation model: Overview}

A core component of this study is the development of a microscopic simulation model that seamlessly integrates both transit and traffic movements on a selected subnetwork in downtown Toronto. The model is developed using Paramics, a suite of high performance software tools for microscopic simulation of realistic traffic networks.

The developed integrated model enables

- capturing the dynamics of supply, in terms of the detailed configuration of the transportation network and its performance in response to varying demands and control

- capturing the dynamics of demand, in terms of dynamic user behavior in response to observed supply, either directly or via traveler information systems

- capturing the complex dynamic interaction between supply and demand

In the model, individual vehicles are represented in fine detail for the duration of their entire trip, providing accurate traffic flow, transit time, and congestion information, as well as enabling the modelling of the interface between drivervehicle units and transit priority.

\section{Approach motivation: The general role of microsimulation in dynamic transportation management}

The prime objective of using traffic simulators is for dynamic transport management. More specifically, simulators can play two distinct roles: (1) an off-line evaluation/design tool and (2) an on-line control-guidance tool. Both roles cover numerous applications such as the provision of trav- eler information and route guidance, a wide variety of surface street and freeway adaptive control measures (including signal priority), incident management, automated toll applications, assessment of the environmental impact of transport design, and management, to name a few.

Transportation networks are, by default, dynamic systems that exhibit continuous changes in both supply-performance and demand. Unexpected events, incidents for example, inevitably occur and therefore change the supply side of the network. Any intervention by the Traffic Management Centre (TMC) in charge, possibly in the form of updating control measures, further changes the supply side. Such changes motivate drivers to change their behavior in several ways, including pre-trip and (or) en-route route choice variations, either within the day, or from day to day, giving rise to dynamic demand profiles.

The choice of such an integrated approach for modelling the 504 King streetcar line should be evident based on the above condensed conceptualization of dynamic transportation network management. The prime motivation, in summary, is "fine details of dynamics".

\section{Base case scenario: Data, development, and calibration}

\section{Data types and sources}

The data used in this study were obtained from a variety of sources. Various sections of the Toronto Works and Emergency Services Department provided the Toronto centreline data, base plans, traffic signal data, and turning movement counts. Transit-related information such as routes, schedules, streetcar operations, stop locations, passenger boardings and alightings, transit vehicle specifications, as well as speed and delay data were provided directly by the TTC. Further data were collected on a number of site visits, particularly related to intersection layouts, turn restrictions, and complianceviolation rates. All data pertain to the morning peak period only.

First, a skeleton network was built, which defines the position of the main nodes and links in the model. This is achieved by an AutoCAD ${ }^{\circledR}$ (DXF) file that is displayed within Paramics, to function as an overlay for manual network coding. It was necessary to import the file into ArcInfo and discard superfluous display layers. From ArcInfo, it was then possible to export the file to the DXF format.

The accuracy of the microsimulation model is generally improved by replicating the roadway geometrics as closely as possible. To provide more detail, base plans for the King Street corridor were also used. These are MicroStation ${ }^{\circledR}$ digital files based on the property data sheet series, which is maintained by Survey and Mapping Services. The Topographic Mapping section of Survey and Mapping Services provided assistance by converting the MicroStation ${ }^{\circledR}$ files to AutoCAD ${ }^{\circledR}$ format. At the same time, they projected the files from the default MTM projection to UTM NAD 27.

\section{Transit line coding}

The 504 King streetcar route was modelled using two separate transit routes: the eastbound route from the Dundas West subway station via Roncesvalles, King, and Broadview 
Fig. 1. The King streetcar route.

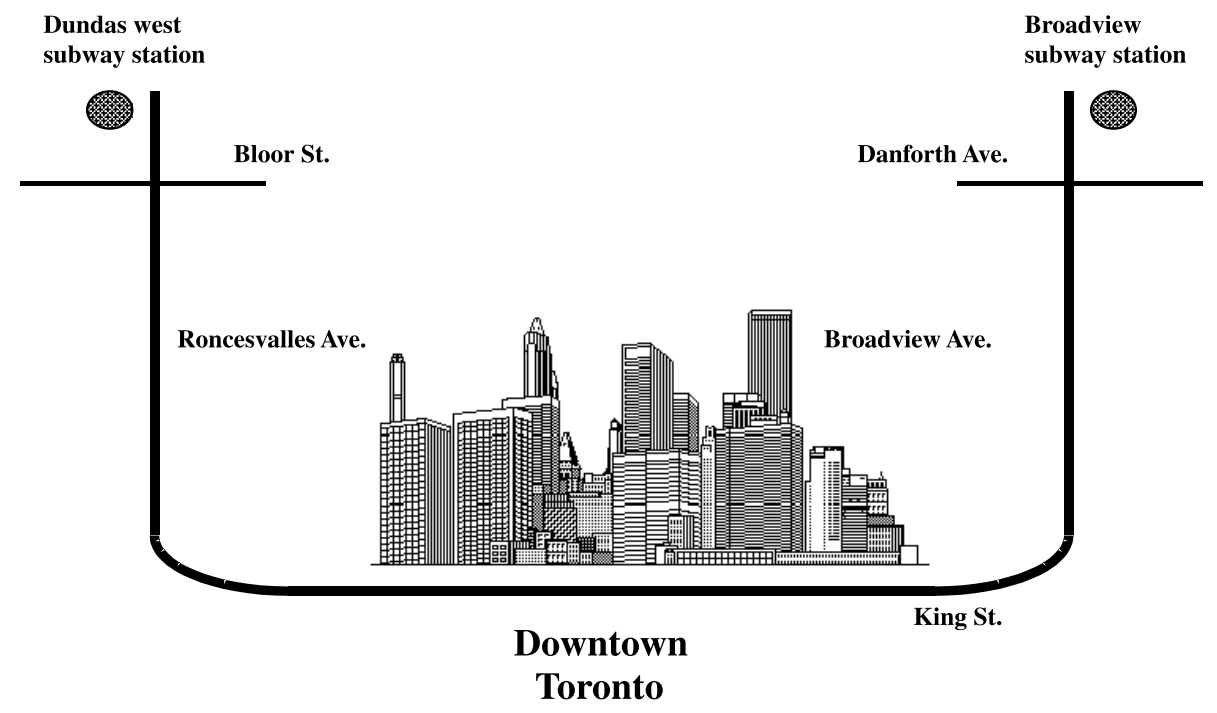

to the Broadview station, and the westbound route from the Broadview station to the Dundas West station.

The King streetcar travels in the centre lane in mixed traffic. This was replicated within the model using lane restrictions. In this case, the streetcar vehicle type was banned from the curb lanes to force the streetcars to use only the centre lane. For the most part, other vehicles were permitted to use all lanes, to emulate the mixed traffic conditions.

Two sections of King Street have designated streetcarexclusive lanes: from Dufferin Street to John Street, and from Jarvis Street to Parliament Street The restrictions are in effect from 0700 to 0900 and from 1600 to 1800 , Monday to Friday.

However, the compliance rate along the two sections is not very high in reality. Simply using lane restrictions to ban all vehicles except for streetcars from the centre lane would not be realistic, as this would be reproducing a $100 \%$ compliance rate. Therefore, the general vehicle population was divided into two groups, with $50 \%$ being in a vehicle type that was banned from the exclusive streetcar lanes and the other $50 \%$ not being restricted.

Many of the nonrestricted vehicles continued to use the curb lane in free-flow conditions, and a higher percentage would select the lane designated as "streetcar exclusive" if the curb lane experienced congestion. The resulting behaviour was quite realistic, with an actual compliance rate of approximately $70 \%$. In discussions with the City of Toronto and the TTC staff, this was seen as a reasonable representation of reality.

The streetcar schedule was based on information provided by the TTC, and streetcars were released from the origin stations at the (fall 2000) peak headway of 2 min in each direction. Transit speed and delay data including travel time from origin to destination, passenger service time, signal delay, congestion delay, and trip speed were provided by the TTC. This speed and delay survey was performed from 0730 to 0830 on 30 November 2000.

\section{Modelling streetcar stops}

There are a total of 114 streetcar stops on the 504 King route. Most of these stops are located at the near-side of in- tersections. The exceptions are three stops on the eastbound route and four stops westbound, which are located at the farside with streetcar platforms. The TTC provided information on stops, including the exact location of the stops in terms of distance from the cross-street curb, as well as distance from the previous stop.

Proper modelling of the streetcar stops was a critical part of this work, because streetcars have different operational characteristics from other transit vehicles. Paramics, as is the case with many other microsimulation packages, does not have the built-in capability to properly reproduce the behaviour of streetcars at stops in mixed traffic, particularly stopping in the centre lane and forcing all traffic in adjacent lanes to stop behind the streetcar while serving passengers.

One viable solution was proposed, used in this study, and shown in Fig. 2. Artificial traffic signals were implemented at all streetcar stops in the model. For this purpose, additional nodes were added at the streetcar stop locations, and traffic signals could then be associated with these nodes. The artificial signals force streetcars to stop by providing a red phase, according to the calculated passenger boarding and alighting time. The artificial signals in the model are actuated signals and are controlled by loop detectors that are programmed to only detect streetcars. A special programming language for actuated signals is available within the base Paramics Modeller software module and was used to implement these special streetcar-stop signals.

The streetcar-specific detectors are usually located $10 \mathrm{~m}$ before the artificial signals. This ensures that, under freeflow conditions, the streetcar will have enough reaction time to be able to stop after being detected at the special streetcar detector and calling the red phase at the artificial signal. In addition, if other vehicles are queued ahead of the streetcar at the adjacent crossroad and the streetcar cannot advance all the way to the proper streetcar stop location, it will nevertheless be able to service passengers as soon as it has reached the streetcar-specific detector. In other words, streetcars will let passengers board and alight up to $10 \mathrm{~m}$ back of the proper stop location if other vehicles are queued ahead. This was found to be a reasonable reproduction of actual conditions, as discussed with TTC staff and observed in the field. 
Fig. 2. Modelling streetcar stops.

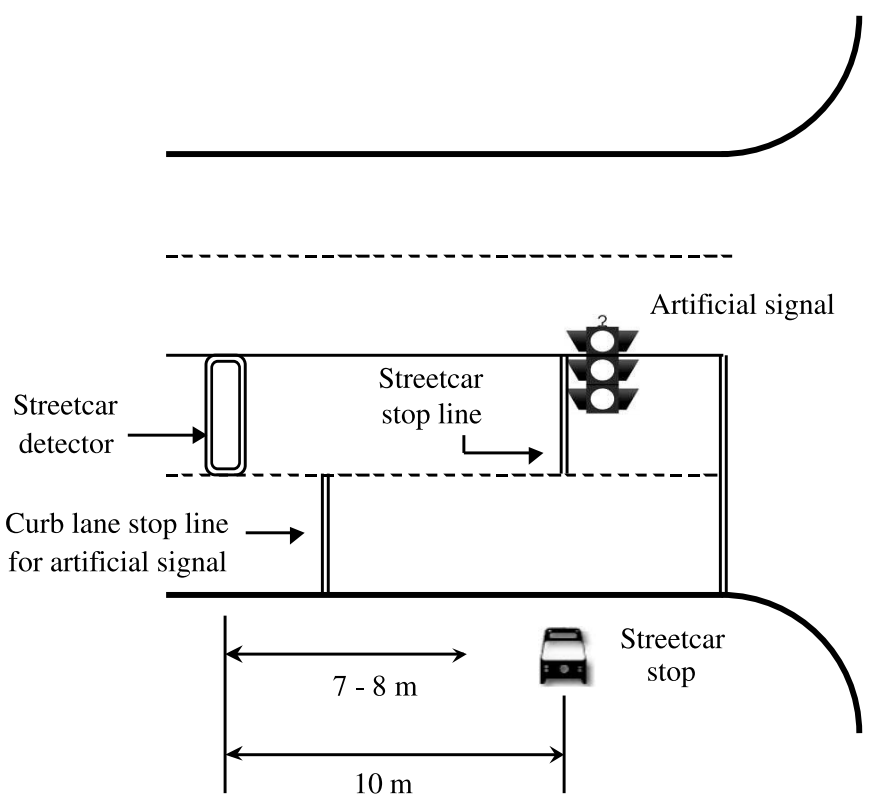

In the curb lane, the stop line associated with the artificial signal is 7-8 m behind the streetcar stop location, forcing vehicles to stop approximately half-way back along the streetcar while passengers are boarding and alighting at the stop. Figure 2 shows a typical near-side streetcar stop with an artificial signal.

The TTC also provided data for passenger boarding and alighting counts for each stop. These statistics were collected from 0800 to 0900 on 10 January 2000 and were the most recent values available for this study. The passenger counts were used to implement the artificial signals at streetcar stops.

Once a streetcar activates the special loop detector, the artificial signal will provide a red phase to this roadway direction for the calculated dwell time. The default boarding time at a streetcar stop was set to $3 \mathrm{~s}$ per passenger, with an additional $4 \mathrm{~s}$ per stop. The default alighting time was set to $2 \mathrm{~s}$ per passenger, and $3 \mathrm{~s}$ of extra time. The actual streetcar dwelling time at a stop will be the maximum of the calculated boarding time and the calculated alighting time.

The passenger boarding time at a stop will have variable values, depending on the actual headway from the previous streetcar. Therefore, a streetcar that has arrived after a longer headway will require more time to board passengers.

\section{Intersection coding}

The 504 King streetcar route traverses 32 signalized and 80 unsignalized intersections. The lane usage at intersections, turning restrictions, and traffic signals were coded at this stage. At unsignalized intersections, every turning movement is assigned a priority of major, medium, minor, or barred. Traffic flows at signalized intersections use the same major, medium, minor, or barred designation, but the designation for each turning movement can change with each of the phases that are offered at a signal.

The modelling of actuated signals was executed in the Paramics plan language, which is similar to a $\mathrm{C}$ programming language. The plan language associates particular detectors with specific signals and defines the control parameters for changing the signal settings. A single signal plan can be used for a number of intersections that have the same signal control algorithm, yet have their own parameters such as minimum green and maximum number of extensions. However, a different signal plan must be defined for every unique signal algorithm.

For each of the 32 signalized intersections in the network model, the Toronto Traffic Signal Control Section provided an MTSS intersection timing report, as well as a timing card. The timing report provides such information as control mode, controller type, transit preemption type, transit preemption interval, number of detectors, timing plans and schedules, intervals, aspects, cycle lengths, offsets, minimum green times, and extensions. For many of the intersections that feature transit signal priority, an additional document was provided, with a written description of the existing priority algorithms.

\section{Transit pre-emption}

The 504 King Street route corridor was the first location where transit signal preemption was implemented in Toronto. As of fall 2000, transit signal preemption was active at 27 of the 32 signalized intersections in the study area.

At an intersection with transit signal priority, if a streetcar has been detected at the upstream "request" loop (approximately $80 \mathrm{~m}$ from the stop line) and has not yet crossed the "cancel" loop at the stop line, the controller considers the "zone" to be "active" for this transit route direction. Two basic algorithms are used to provide signal priority for transit vehicles: transit-corridor green extension, and cross-street green truncation. In case of any disruption to the offsets (in reference to the master system clock) by the provision of signal priority an offset recovery routine is initiated.

If either of the "zones is active" (i.e., for either transit route direction) at the time of the decision point for transitcorridor green extension, the green extension algorithm will begin with an initial fixed green time period for the transit corridor. This is followed by demand-dependant extensions (1 or $2 \mathrm{~s}$ depend on controller types) for the transit-corridor green. The extensions are served consecutively until the zone is cleared (i.e., streetcar passes the cancel loop) or until a maximum number of extensions are provided.

An additional decision point is defined for the truncation. If the zone is active at the time of this decision point, the signal will also switch to local control. The signal timing will be altered to shorten the cross-street green time and hasten the provision of green to the transit corridor. The amount of green time that will be subtracted from the cross street is a set value defined per intersection, ranging from 2 to $6 \mathrm{~s}$ after minimum walking time in the study area.

Those decision points for transit-corridor green extension and cross-street green truncation are defined per intersection after pedestrian walking time and signal priority can be provided in successive cycles if the "zone" is still active.

This offset is in reference to the master system clock, and is valid for the beginning of the first interval. If the actual offset is up to a quarter of the cycle length behind the proper offset, offset recovery can occur by shortening the transition intervals to the minimum. In addition, the total amount of time subtracted during a cycle may not be more than one eighth of the regular cycle length. The algorithm will also 
check if this method (shortening green) would take more than two cycles to return to the proper offset: if this is the case, the lengthening method will instead be chosen.

More details of the above routines can be found in Abdulhai et al. (2001).

\section{Traffic demand: Developing the origin-destination matrix}

The simulation model represents the traffic and transit operations in the study network during the morning rush hour on a typical weekday of fall 2000. As such, the input traffic demand should represent the same time period. However, the only available source of origin-destination trip data is the Transportation Tomorrow Survey (TTS), the most recent of which (at the time of the study) was conducted in 1996. Obviously, these data are inconsistent with the desired simulation of fall 2000 conditions. In addition, link and turn volumes in a sub-area resulting from the assignment of an O-D matrix to the road network using regional transportation models such as EMME/2 may not necessarily match the actual real-world traffic counts for the same sub-area, even if the O-D matrix and the traffic counts were for the same analysis time period. Therefore, this study used a "reverse traffic assignment" approach to adjust the relevant 1996 OD matrix to represent the actual fall 2000 traffic conditions. In contrast to the conventional traffic assignment which assigns an input O-D matrix to the road network producing link and turn volumes, this approach uses actual link and turn volumes (in our case, for fall 2000) to adjust the input O-D matrix (in our case, the 1996 O-D matrix), in an iterative process involving several traffic assignments, so as to cause the new adjusted O-D matrix to reproduce the actual observed volumes, if assigned to the road network. This approach has been implemented in our study using the gradient-based model developed by Spiess (1990). The model is formulated as a convex minimization problem, assuming nondecreasing link cost functions to ensure convexity of the model. The resulting $R^{2}$ (measuring how close the traffic movement volumes from the procedure are to the actual traffic volumes) was 0.853 , which compares favourably with other reported results (Spiess 1990). Figure 3 shows the actual vs. the assigned volumes. The updated O-D matrix from EMME/2 was then input into Paramics. Even though there are relatively large differences between actual and modelled counts of a few traffic movements, the developed O-D matrix was used in the simulation model without further improvements because it replicated the effects of traffic congestion on streetcar operations adequately, as indicated in Table 1.

\section{Simulation and calibration}

The simulation model was developed for the morning peak. The total time period simulated within Paramics was $3 \mathrm{~h}(0630-0930)$. The first $30 \mathrm{~min}$ of simulation time were a warm-up period to fill the network with vehicles and streetcars. No statistics were gathered for these $30 \mathrm{~min}$.

Data were actually collected for the simulation period representing 0700 to 0930 . A fairly long simulation time was required so that a significantly high number of streetcars could complete a full trip, even under adverse conditions or scenarios.
Each microsimulation run is actually a random experiment. Therefore, numerous runs were required for each scenario and also for all interim and test networks that were necessary, yet are not documented in this report. This is required to ensure the validity and stability of the results.

As with every modelling exercise, a process of calibrating the base case network was necessary. Within microsimulation there are two types of calibration; the first is geometric network calibration. This consists of running the initial network and looking for any unusual behaviour or results that may be due to inexact coding of the network. This can be done visually, by observing the individual vehicles, signals, etc., via the Paramics graphical user interface. In addition, a variety of statistical outputs can be gathered to support this calibration effort.

Problems that can often be observed include unrealistically long queues, complete network breakdown, or perhaps unreasonably short queues or high speeds. Initially, one must review all the previous coding steps, from basic network construction to traffic signals and transit coding, and identify possible coding errors. Adjustments also include curb and stop line exact locations.

A number of system-wide simulation parameters were also used to calibrate the network, including time steps per second (used 10), aggressiveness and awareness distributions (used uniform distribution), mean headway (used $1 \mathrm{~s}$ ), and mean reaction time (used $1 \mathrm{~s}$ ).

Numerous runs with various seed values were completed in all cases to ensure the validity of the results. Table 1 shows the comparison of travel time and speed of streetcars between the calibrated and real networks. Eleven signalized intersections were chosen for the purpose of comparing turning vehicle counts. As shown in Table 2, the results show good correspondence between the calibrated and real networks.

\section{Scenario development and discussion of results}

\section{Scenarios}

The developed model is very flexible and can in fact be used to examine a wide array of operational scenarios. In this effort, we focused on four most pressing scenarios, which are as follows:

Base case: representing the status quo of streetcar operations on the King line, including the already existing transit signal priority system.

Scenario 1, No signal priority: the same as the base case except the signal priority system is turned off. Comparison of this scenario with the base case would be indicative of the merits of the already deployed priority system.

Scenario 2, Base case with banning all left-turn vehicles: impedance to streetcar progression through signalized intersections is partly due to left turning traffic, which can block the streetcar track for a significant length of time while waiting for an acceptable gap to cross opposing through traffic. Banning left turn movement is therefore expected to improve streetcar operations along King Street. This scenario would quantify the expected benefits of such measure.

Scenario 3, Base case with banning all vehicles from King Street: the most aggressive scenario examined in this study 
Fig. 3. Assigned vs. observed volumes.

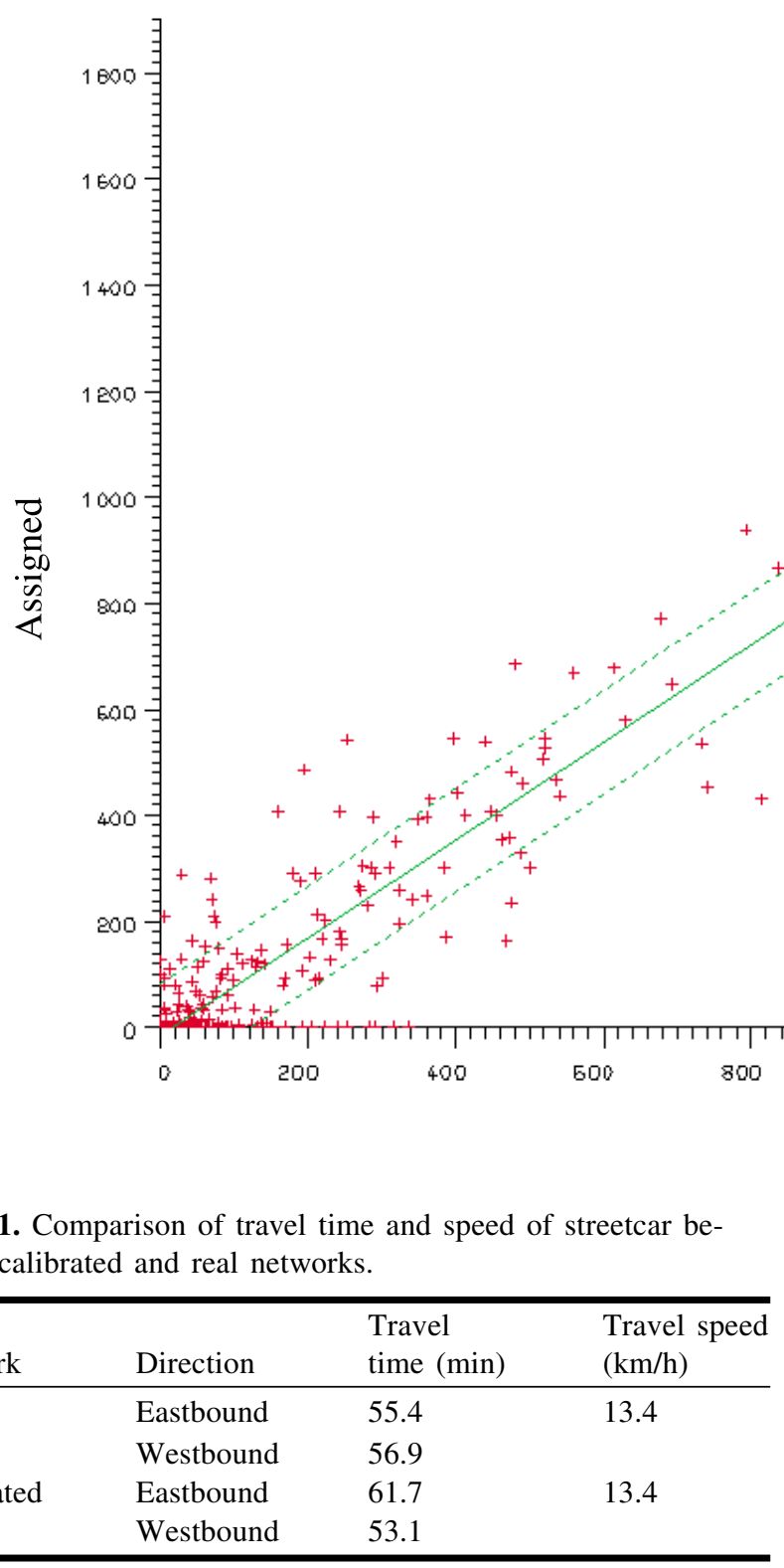

Table 1. Comparison of travel time and speed of streetcar between calibrated and real networks.

\begin{tabular}{llll}
\hline Network & Direction & $\begin{array}{l}\text { Travel } \\
\text { time }(\mathrm{min})\end{array}$ & $\begin{array}{l}\text { Travel speed } \\
(\mathrm{km} / \mathrm{h})\end{array}$ \\
\hline Real & Eastbound & 55.4 & 13.4 \\
& Westbound & 56.9 & \\
Calibrated & Eastbound & 61.7 & 13.4 \\
& Westbound & 53.1 & \\
\hline
\end{tabular}

is to ban traffic entirely from King Street to ensure uninterrupted flow of streetcars along the King route. This scenario examines and quantifies the expected benefits. It should be noted, however, that this scenario would not necessarily indicate the adverse effect of traffic on parallel roads (for instance, traffic diverting onto Queen Street instead). The model used in this study is focused solely on King Street, and therefore no parallel arterials are modelled. Later expansions of the model could include several parallel arterials and give King traffic some rerouting options to assess the diversion impacts.

For scenario 2 (i.e., left turn prohibition), Paramics automatically eliminates the zone-to-zone trips if they do not have any appropriate route between origin and destination. Therefore, all zone-to-zone trips that have no options but to make a left turn were set to zero. This caused about $10 \%$ reduction in the total number of trips. To compensate for such an artificial reduction caused by the nature of the

\section{Observed}

model, $10 \%$ of total demand was added before the elimination of left-turning trips. The overall level of "traffic usagecongestion" was therefore restored to normal-typical.

For scenario 3 (i.e., full traffic banning), all traffic destined to any zone on King Street were set to zero. Vehicles are allowed only on Roncesvalles and Broadview Avenues in this scenario. The only vehicle type granted access to King Street is streetcars.

\section{Measures of effectiveness}

To assess the relative impact of the above scenarios, the following set of measures of effectiveness was used:

- Cycle time: the time for a streetcar to complete a round trip, both in absolute minutes and as a percentage change relative to the status quo.

- Average transit speed in kilometres per hour.

- Average headway: the average time gap in seconds between successive streetcars, taken at all 106 stops along the route. Note that the release headway of streetcars from the terminal was fixed at 2 min for all scenarios. However, for each scenario, the actual headways along the line vary because of a combination of interrupting factors (i.e., if there was no interruption, streetcars would arrive at each stop exactly every $2 \mathrm{~min}$ ). Therefore, the average headway (measured for 106 stops) captures the effective headway for the entire route under the considered scenario.

- Headway standard deviation, indicating the variability around the mean headway. 
Table 2. Comparison of turning vehicle counts between calibrated and real networks.

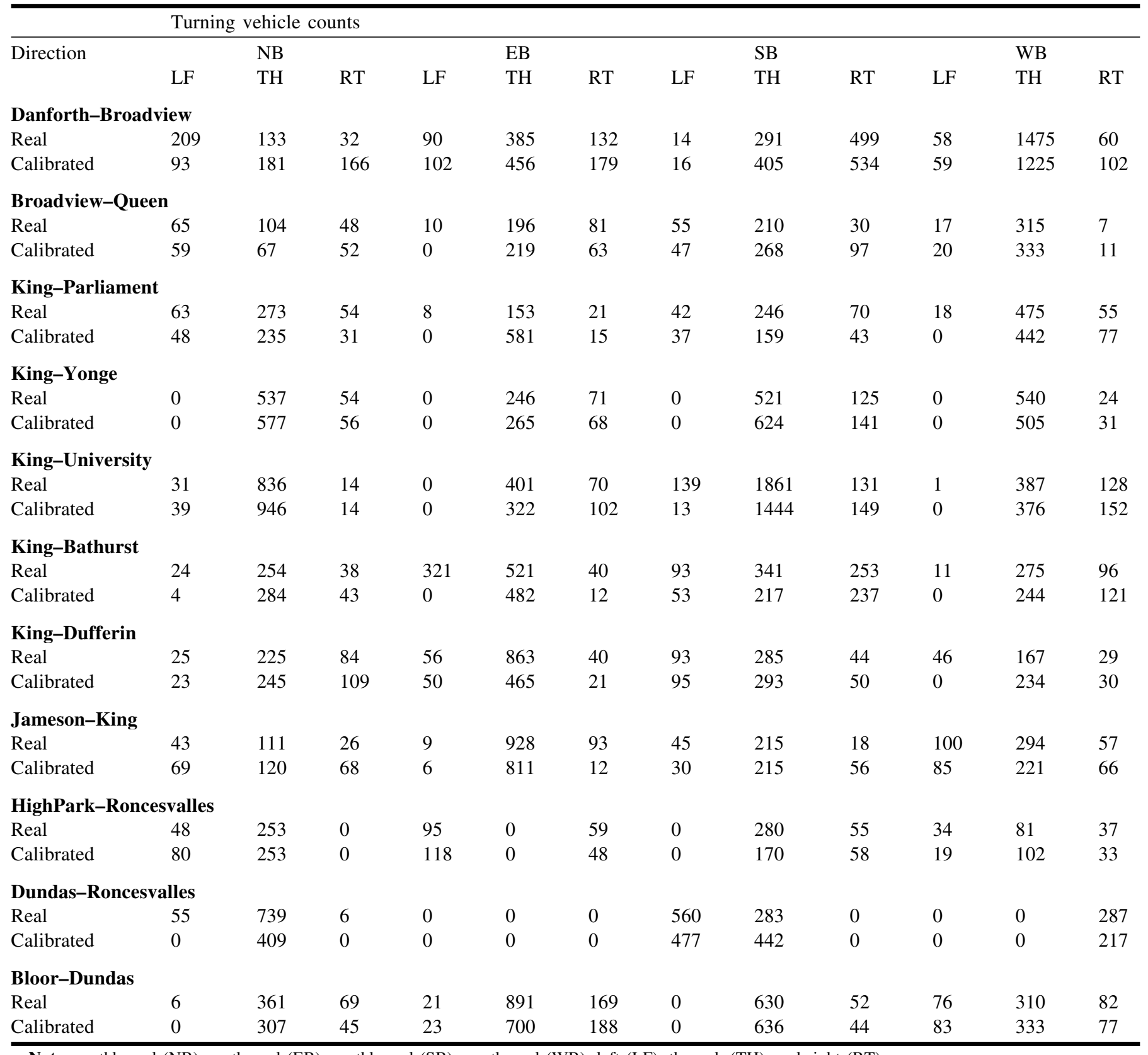

Note: northbound (NB), eastbound (EB), southbound (SB), westbound (WB), left (LF), through (TH), and right (RT).

- Average service frequency in streetcars per hour: the reciprocal of average headway, i.e., frequency $=1 /$ headway, defined as the average streetcar flow (bearing in mind that the schedule headway is fixed at 2 min for all scenarios, as discussed above).

- Person throughput: defined as the service frequency multiplied by an assumed average peak-period vehicle load of 75 passengers.

- Transit vehicle bunching: loosely interpreted as the percentage of transit vehicles following too close to each other (i.e., with very short headways). To quantify the above subjective measure, we report once on the percentage of headways less than $60 \mathrm{~s}$ and another on the percentage of headways less than $30 \mathrm{~s}$.
- Fleet size: the number of streetcars serving the route. Given that fleet size = cycle time/headway, any improvement in transit cycle time and (or) frequency results in a reduction in the required fleet size. Therefore, the change in fleet size is the change in the number of transit vehicles required to serve the line.

- Overall average speed including transit as well as traffic in kilometres per hour.

\section{Results}

Figure 4 shows the performance summary based on the above measures of effectiveness. For the absolute transit cycle time in minutes and the percentage change in transit cycle time relative to the status quo, it is evident that the 
current implementation of transit priority (status quo) is beneficial to transit operations along the line relative to the case where no priority is assigned to transit. Termination of transit priority assignment would result in $11 \%$ increase in transit cycle time. Left turn banning shows significant potential to further reduce transit cycle time by $13 \%$. Total traffic ban on the route, no doubt, shows potential reduction in roundtrip time by $30 \%$ over the base case.

For transit speed in kilometres per hour, the figure indicates a systematic upward pattern of speed due to the addition of transit priority, left turn banning, and exclusive dedication of King Street to transit. It is notable that transit priority alone is less effective compared with the additional left turn banning, which is consistent with expectations that streetcar travel time savings at traffic lights due to priority could very well be diminished while it is stranded behind a left turning vehicle waiting for an acceptable gap.

For average headway in seconds, and opposite to speeds, the results show a systematic downward pattern in average headways due to the addition of transit priority, left turn banning, and exclusive dedication of King Street to transit. It is notable that the "No Traffic" scenario results in a $122 \mathrm{~s}$ average headway, which is very close to the schedule headway of $120 \mathrm{~s}$. Again, and for the same reasons as above, it is evident that transit priority alone without left turn banning has the least effect.

Equally important is the headway standard deviation, which indicates the variability around the mean headway. From a transit rider perspective, the unpredictability of the streetcar arrival is the most frustrating. The results show that transit priority alone is effective in this regard, with only marginal improvement due to left turn and full traffic prohibition, respectively. It should be noted that the standard deviations for the base case, no left turn, and no traffic scenarios are still significantly high compared with the corresponding average headways, indicating a fairly spread distribution of headways, as shall be seen later.

Figure 4 also shows the average service frequency, measured in transit vehicles per hour. This is the mirror image of average headway. The figure shows minor changes in service frequency due to signal priority alone, but higher frequencies can be achieved because of banning of left turns (1.7 more streetcars per hour over the base case) or traffic banning (almost 3 more streetcars per hour over the base case).

It should be noted again that the frequencies reported here are the "effective" frequencies, given constant release headway (at the terminal) of 1 streetcar every 2 min (i.e., 30 streetcars per hour). It is implied here that the vehicle fleet size (i.e., number of required streetcars to serve the route) is not fixed but in fact is reduced for each scenario (assuming a fixed release headway of $2 \mathrm{~min}$ ), mainly because of the reduction in cycle time. This will be discussed shortly. It is conceivable that higher frequencies can be achieved by reducing the schedule headway lower than 2 min for the no left turn and no traffic scenarios but with further operations control and other methods such as proof of payment to reduce dwell time. Headways of $1 \mathrm{~min}$ (or even shorter) have been reported in the literature.

The pattern for person throughput is similar to that of service frequency. In fact, it is exactly the same, only converted to passengers per hour via multiplying by a constant of 75 passengers per vehicle during the peak period.

The figure also reports on the percentage of headways less than $30 \mathrm{~s}$, which is an arbitrary threshold chosen to define very short headway as an indication of the phenomenon known as "bunching". Bunching is a critical problem in transit operations, and results need careful interpretation. It is clear that both transit priority and further banning of left turns reduce the level of bunching, which is also intuitive. It seems, however, that full traffic prohibition could increase bunching. One possible explanation could be that vehicular traffic on the route acts like a "cushion", separating streetcars. In the absence of such a cushion, once a streetcar is interrupted, say at a traffic signal, the streetcars behind catch up with it, which is obviously not the case if there is traffic between the two. It should be noted that despite transit priority, some streetcars get caught at some intersections, as priority assignment (or not) depends on the streetcar arrival time at the priority call detector relative to the traffic light cycle. It should be noted that in the case of full traffic ban, increased bunching is not necessarily in contradiction with this scenario having the lowest headway standard deviation, as discussed earlier. Frequency distributions of the headway for the four scenarios (not shown for space limitations) indicate that the no traffic scenario has a headway distribution with higher percentages of headways less than $30 \mathrm{~s}$ compared with the other distributions because the distribution itself for that scenario is "tighter" and shifted to the left compared with the other scenarios. This seemingly negative impact, however, should not be taken in isolation of the rest of results, such as improvement in speed, headways, and so on. It is also important to note that no operational control methods were modelled here. With no traffic interference in the no traffic scenario, such control methods can effectively reduce bunching and improve schedule adherence.

Fleet size is defined as the number of cars that are required to serve the line. Given the simple relationship that fleet size = cycle time / headway, any improvement in transit cycle time and (or) headway results in a reduction in the required fleet size. Figure 4 indicates that an almost $30 \%$ reduction in fleet size is achievable by the implementation of a transit mall on King Street, relative to the no priority case. Given the cost of acquiring and maintaining streetcars, it seems plausible to adopt approaches that would reduce the required fleet size as shown.

Finally, Fig. 4 also shows the overall average speed including transit as well as traffic in kilometres per hour. This figure and related numbers should also be analyzed with caution because of possible misinterpretation. It shows that the implementation of transit priority causes a slight drop in overall combined average speeds, as the gain in transit speed is associated with a loss for vehicular traffic. Banning left turns significantly improves all speeds on King Street, as a stranded left turning vehicle blocks not only streetcars behind it but also the rest of the traffic. Therefore, banning left turn is a viable option not only for transit but for traffic as well. Once traffic is fully prohibited to access King Street further improvements in speeds take place, which might be counter-intuitive. However, this seems to be more a modeling artifact than real. Owing to the absence of alternate routes in the model, banned traffic "disappears" from not 
Fig. 4. Performance summary: $(a)$ cycle time in minutes, $(b)$ cycle time as percentage of base case, $(c)$ transit speed in kilometres per hour, $(d)$ average headways in seconds, $(e)$ headway standard deviation, $(f)$ average service frequency vehicle/h, $(g)$ passenger throughput passenger/h, $(h)$ bunching: \% headways $<30 \mathrm{~s}$, $(i)$ fleet size (number of cars), and $(j)$ average traffic and transit speeds in kilometres per hour.

(a)

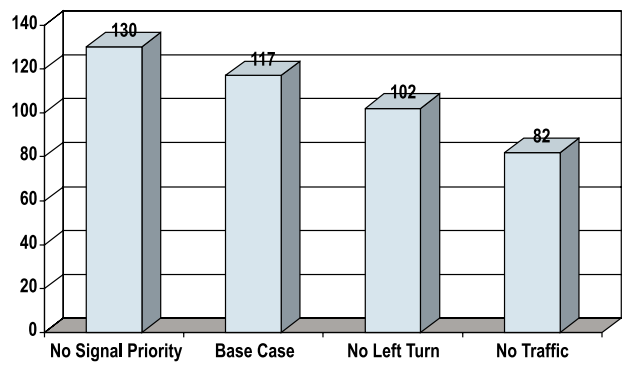

(c)

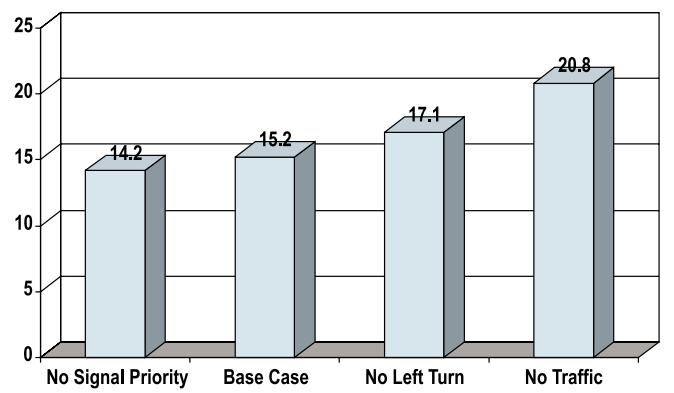

(e)

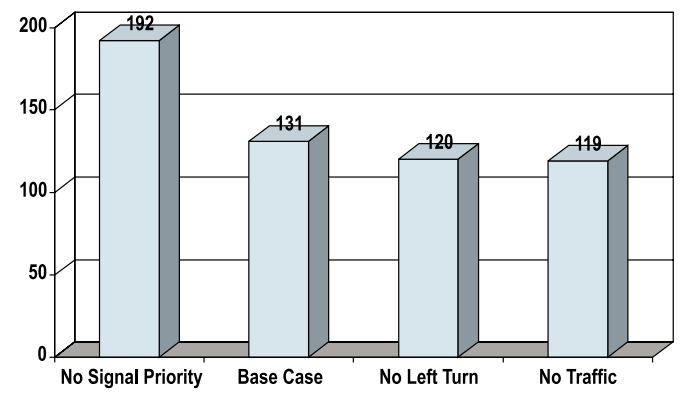

(g)

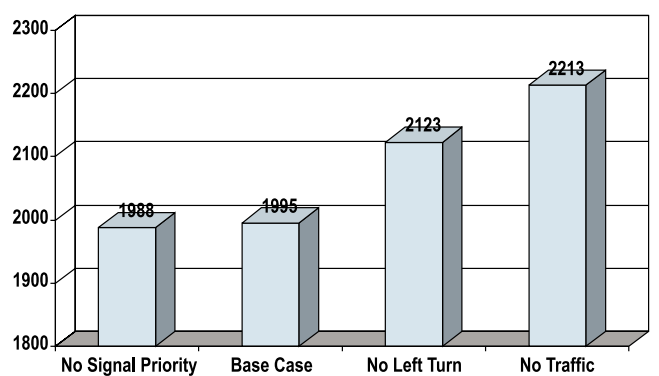

(i)

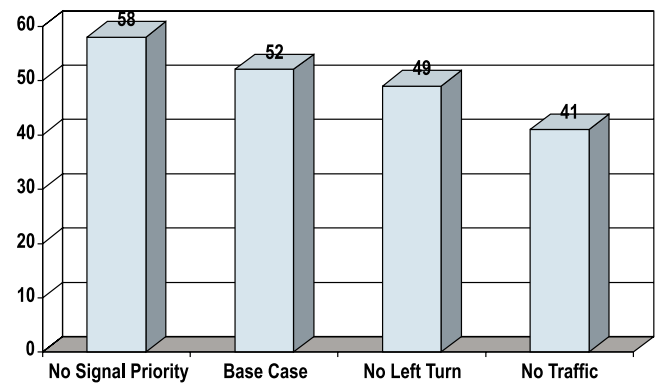

(b)

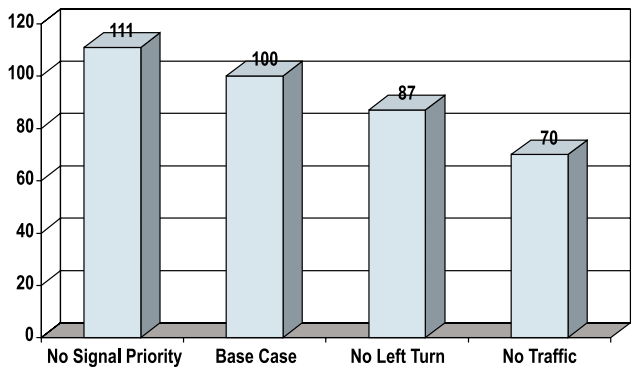

(d)

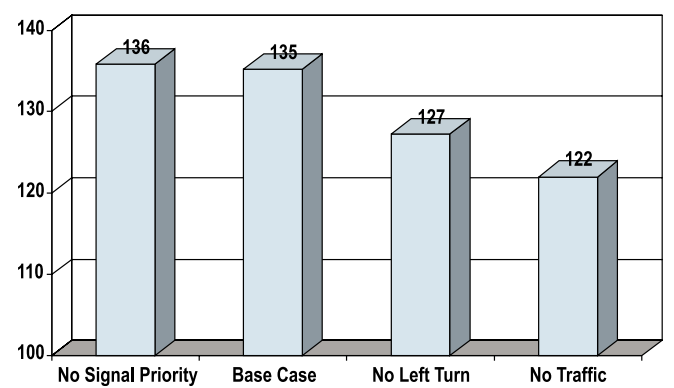

(f)

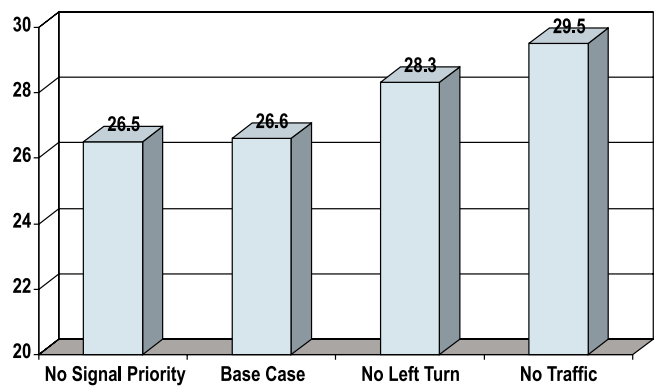

(h)

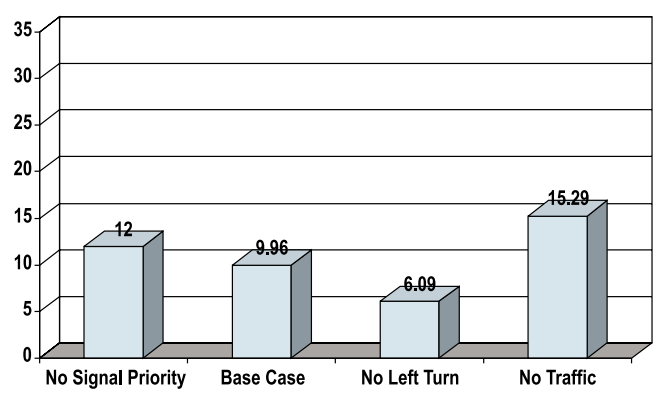

(j)

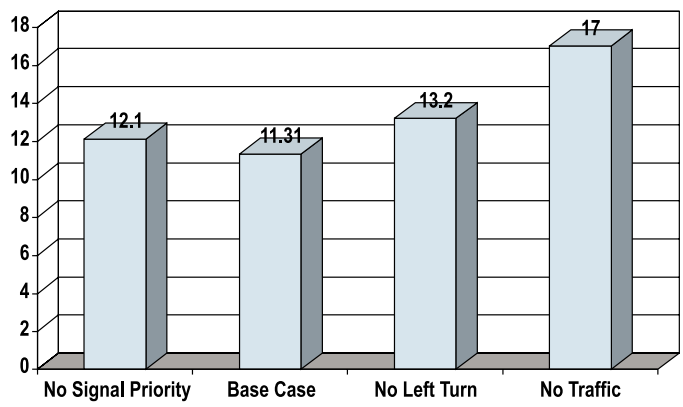


only King Street but from the entire network, leaving only faster streetcars in the network. In reality, this traffic would actually divert to parallel routes, causing travel time increase on such routes and potentially appearing as cross traffic on King Street intersections causing some delays to streetcars. The only way to capture the true effect on traffic, therefore, seems to be via network expansion to include several parallel routes north and south of King Street.

\section{Summary and conclusions}

This study is focused on quantifying the impact of adopting transit priority schemes, with streetcar operation along King Street in the heart of Toronto as a case study. The study relied on developing an integrated traffic and transit microsimulation model to analyze the four most pressing scenarios, which are the status quo including existing transit priority, the no transit signal priority case, the prohibition of all left-turning traffic case, and finally the full traffic ban case. To quantify the impact of any of the above scenarios, a set of common measures of effectiveness was used as detailed above including transit travel time and speed, headways, service frequency and person throughput, bunching, fleet size implications and overall traffic, and transit average speeds.

Based on the integrated microsimulation model results outlined in this report, the following conclusions can be drawn:

- The current implementation of transit priority (status quo) is beneficial to transit operations along the line relative to the case where no priority is assigned to transit. Termination of transit priority assignment would result in $11 \%$ increase in transit cycle time (i.e., round trip time). Left turn prohibition shows significant potential to further reduce transit cycle time by $13 \%$. Total traffic ban on the route shows potential reduction in round-trip time by $30 \%$.

- A systematic uprising pattern in transit speeds was observed that is attributable to the addition of transit priority, left turn banning, and exclusive dedication of King Street to transit, in order.

- Opposite to speeds, a systematic downward pattern in average headways due to the addition of transit priority, left turn banning, and exclusive dedication of King Street to transit was also observed.

- A clear systematic downward pattern in headway standard deviation was observed, which is as important as the observed reduction in headways themselves if not more important from a transit rider perspective.

- Very minor improvement of average service frequency is attributed to transit priority alone. However, improvement becomes more significant when left turn or traffic banning is in effect.
- The trend in person throughput improvements is similar to that of the average service frequency. In fact, it is exactly the same, only converted to passengers per hour via multiplying by a constant of 75 passenger per vehicle during the peak period.

- It is clear that both transit priority and further banning of left turns reduce the level of bunching. It seems, however, that full traffic prohibition could increase bunching because of the lack of what we term as "traffic cushioning" between streetcars when traffic is permitted. This does not contradict the improvement in headway standard deviations, because a less spread headway distribution with lower mean is compressed and shifted to the left (lower headways) relative to the other cases, thus having a higher percentage of headways below any chosen cut-off limit.

- An almost $30 \%$ reduction in fleet size is achievable by the implementation of a transit mall on King Street, relative to the no priority case.

- Full traffic prohibition seems to improve overall combined traffic and transit speeds, which we believe to be more of a modelling artifact than true performance as explained above.

Finally, one could argue on the basis of obtained results that banning left turns and the transit mall options are more or less equal in terms of benefits, while the latter is much more expensive to implement in terms of cost and potential public opposition. Therefore, the transit authorities are encouraged to try the left turn ban first and experiment with different levels of enforcement before attempting the transit mall option.

\section{Acknowledgements}

The authors are thankful to the City of Toronto, Communication and Information Technology (CITO), Natural Sciences and Engineering Council of Canada (NSERC), and the Toronto Transit Commission (TTC) for their cooperation on this study. Their funding as well as in-kind support made this research possible.

\section{References}

Abdulhai, B., Shalaby, A., Lee J., and Georgi, A. 2001. Assessment of transit priority impacts for the 504 street car route: An integrated transit and traffic microsimulation approach. Transportation Planning, City Planning Division, Urban Development Services, City of Toronto, Toronto, Ont.

City of Toronto. 2001. A transportation vision for the City of Toronto official plan. Transportation Planning, City Planning Division, Urban Development Services, City of Toronto, Toronto, Ont.

Spiess, H. 1990. A gradient approach for the O/D matrix adjustment problem [online]. Available from http://www.spiess.ch/ emme2/demadj/demadj.html [accessed 14 August 2003]. 